\title{
At therapeutic concentration bupivacaine causes neuromuscular blockade and enhances rocuronium- induced blockade
}

\author{
Ji Hyeon Lee, Soo-Il Lee, Seung Cheol Lee, So Ron Choi, and Won Ji Rhee \\ Department of Anesthesiology and Pain Medicine, Dong-A University College of Medicine, Busan, Korea
}

Background: Partially paralyzed patients may be placed in the risk of pharyngeal dysfunction. Bupivacaine acts as acetylcholine receptor ion channel blocker and may synergistically interact with rocuronium to augment NM blockade. Thus, this study aims to elucidate whether or not, at a therapeutic concentration, bupivacaine by itself may cause NM blockade and reduce an effective concentration of rocuronium.

Methods: Twenty-two left phrenic nerve-hemidiaphragms (Male SD rats, 150-250 g) were hung in Krebs solution. Three consecutive ST, $0.1 \mathrm{~Hz}$ and one TT, $50 \mathrm{~Hz}$ for $1.9 \mathrm{~s}$ were obtained before drug application and at each new drug concentration. A concentration of bupivacaine in Krebs solution $(\mathrm{n}=5)$ was cumulatively increased by way of 0.01 , $0.1,1,(1,2,3,4,5,6,7) \times 10 \mu \mathrm{M}$. In a Krebs solution, pre-treated with bupivacaine $0(\mathrm{n}=5), 0.1(\mathrm{n}=5), 1.0(\mathrm{n}=5), 10(\mathrm{n}$ $=2) \mu \mathrm{M}$, and then concentrations of rocuronium were cumulatively increased by way of $1,3,5,7,9,12,14,16,18,20$ $\mu \mathrm{M}$. EC for each experiment were determined by a probit. The $\mathrm{EC}_{50}$ 's of rocuronium were compared using a Student's t-test with Bonferroni's correction. Differences were considered significant when $\mathrm{P}<0.05$.

Results: The potency of bupivacaine for normalized TF was $11.4( \pm 1.1) \mu \mathrm{M}$. Below $30 \mu \mathrm{M}$ of bupivacaine, the single twitch potentiation sustained despite the development of tetanic fade and partial inhibition of PTT. Bupivacaine significantly facilitated the NM blockade induced by rocuronium.

Conclusions: Clinicians should be aware that bupivacaine by itself at its therapeutic concentration inhibit NM conduction and enhances rocuronium-induced muscle relaxation. (Korean J Anesthesiol 2012; 62: 468-473)

Key Words: Bupivacaine, Neuromuscular blockade, Rocuronium.

Received: December 21, 2011. Revised: January 20, 2012. Accepted: January 26, 2012.

Corresponding author: Soo-Il Lee, M.D., Ph.D., Department of Anesthesiology and Pain Medicine, Dong-A University College of Medicine, Dong-A University Hospital, 26, Daesingongwon-ro, Seo-gu, Busan 602-715, Korea. Tel: 82-51-240-5390, Fax: 82-51-247-7819, E-mail: silee@ dau.ac.kr

(c) This is an open-access article distributed under the terms of the Creative Commons Attribution Non-Commercial License (http:// creativecommons.org/licenses/by-nc/3.0/), which permits unrestricted non-commercial use, distribution, and reproduction in any medium, provided the original work is properly cited. 


\section{Introduction}

Sundman et al. [1] showed that after recovery to a trainof-four ratio of more than 0.90 , the incidence of pharyngeal dysfunction was $13 \%$, and the majority ( $80 \%$ ) of pharyngeal dysfunction resulted in laryngeal penetration. Thus, bupivacaine may inhibit neuromuscular conduction, and combining it with other drugs, which interrupt the neuromuscular (NM) conduction, would place the patients at risk of muscle relaxation.

Local anesthetics (LAs) primarily inhibit voltage-gated $\mathrm{Na}^{+}$ channels [2]. However, LAs noncompetitively inhibit the ion channel activity of muscle-type nicotinic acetylcholine receptor (AChR) at therapeutic dose $[3,4]$ and ACh synthesis in nerve terminal [5]. There seems to be a few studies conducted on NM blockade created by bupivacaine.

Some investigators indicated that lidocaine failed to show a sparing effect on cisatracurium requirements [6,7]. However, it has been known that some local anesthetics potentiate nondepolarizing NM blockers [8-11]. Bupivacaine and rocuronium are regarded as NM blocker: bupivacaine acts as an AChRion channel blocker, and rocuronium as AChR blocker. Because of their different acting sites, the two drugs may synergistically interact to produce more NM blockade than expected. Based on this premise, it could be hypothesized that bupivacaine enhances rocuronium-induced NM blockade.

Thus, this study aims to elucidate whether or not, at a clinical therapeutic concentration, bupivacaine by itself may induce NM blockade and reduce an effective concentration of rocuronium.

\section{Materials and Methods}

The Institutional Animal Care and Use Committee approved the experimental protocol. All experimental courses followed the Guide for the Care and Use of Laboratory Animals provided by the National Academy of Sciences. Twenty-two male Sprague-Dawley rats (5-7 weeks in age, and 150-250 $\mathrm{g}$ in weight) were anesthetized by a perivertebral injection of propofol $(50 \mathrm{mg} / \mathrm{kg})$ at the lumbar level and then sacrificed. The phrenic nerve and diaphragm were excised together and the left diaphragm with the middle diaphragmatic ligament was separated from the rest of the diaphragm. Left phrenic nervehemidiaphragm preparations were hung in a $20 \mathrm{ml}$ organ bath that had been filled with Krebs solution (118 mM NaCl, $5 \mathrm{mM}$ $\mathrm{KCl}, 2.5 \mathrm{mM} \mathrm{CaCl}_{2}, 30 \mathrm{mM} \mathrm{NaHCO} 3,1 \mathrm{mM} \mathrm{KH_{2 }} \mathrm{PO}_{4}, 1 \mathrm{mM}$ $\mathrm{MgCl}_{2}$ and $11 \mathrm{mM}$ glucose).

The bath solution was maintained at $32^{\circ} \mathrm{C}$ and was continuously aerated with a gas mixture of $95 \%$ oxygen and $5 \%$ carbon dioxide. The $\mathrm{pH}$ of the bath solution was maintained at 7.38 to 7.42. Spent Krebs solution was exchanged with fresh solution $10 \mathrm{~min}$ after the preparation was mounted. The preparation was attached to a force transducer (Model 1030, UFI, Morro Bay, CA, USA) with a stainless steel wire and was allowed to stabilize for $20 \mathrm{~min}$ in the bath. The phrenic nerve, connected to an electrode, was stimulated with supramaximal square wave impulses in duration of $0.2 \mathrm{~ms}$, using a stimulator (Model ML112, ADInstruments Pty Ltd, Bella Vista, NSW, Australia).

The diaphragm muscle was stretched until the maximum output tension was measured after stimulation, followed by another $10 \mathrm{~min}$ for stabilization before each experiment began. Before drug application for a baseline and at each new drug concentrations, there were three consecutive single twitches (ST) at $0.1 \mathrm{~Hz}$ and a $1.9 \mathrm{~s}$ tetanic tension of $50 \mathrm{~Hz}$ recorded, which were then digitized and stored on a Power Macintosh 7100 (Apple Computer Corp, Cupertino, CA, USA) using data acquisition software (MacLab, ADInstruments Pty Ltd, Bella Vista, NSW, Australia).

After baseline tensions were measured, the concentrations of bupivacaine in Krebs solution $(\mathrm{n}=5)$ were cumulatively increased by the way of $0.01,0.1,1,(1,2,3,4,5,6,7) \times 10 \mu \mathrm{M}$, until an $80 \%$ to $90 \%$ reduction in ST was reached.

In order to determine whether bupivacaine would enhance rocuronium-induced NM blockade, Krebs solutions had been pre-mixed with $0(\mathrm{n}=5), 0.1(\mathrm{n}=5), 1.0(\mathrm{n}=5), 10(\mathrm{n}=2) \mu \mathrm{M}$ bupivacaine, and then the concentrations of rocuronium were cumulatively increased by the way of $1,3,5,7,9,12,14,16,18$, $20 \mu \mathrm{M}$ until an 80 to $90 \%$ reduction in ST was reached.

A period of at least $20 \mathrm{~min}$ was allowed to pass to establish a pseudo-steady state of every drug concentration between bath and muscle. Tension measurements were made during the pseudo-steady state and in the drug-free solution after completion of each experiment. The data were analyzed only if the ST tension returned to greater than $90 \%$ of the baseline recording in a drug-free solution.

Mean ST tension was gathered by averaging the three consecutive measurements, and peak tetanic tension (PTT) was measured at $0.2 \mathrm{~s}$ after start of tetanic stimulation. The effect of the drug on ST tension, PTT, and tetanic fade (TF) presented as a percent reduction of baseline for ST tension, and PTT, with a normalized percent increase of TF ( = [\%increase of TF - \%increase of TF in baseline] / [100 - \%increase of TF in baseline]) [12]. The effective concentrations (EC) of bupivacaine, and rocuronium for ST, and TF were determined by probit model. The concentration-response curves of bupivacaine for ST, PTT, and TF, and rocuronium for ST, and TF were plotted.

Potency ratio of ST to TF for each drug was calculated. The interaction between rocuronium and bupivacaine was determined using an interaction index [13]. 
The $\mathrm{EC}_{50}$ 's of rocuronium given with different concentrations of bupivacaine were compared using a Student's t-test with Bonferroni's correction for the significance level. Differences were considered significant when $\mathrm{P}<0.05$.

\section{Results}

Bupivacaine created reversible and concentration-dependent NM blockade. In terms of the influence of bupivacaine on neuromuscular conduction, tensions of ST were equal to or greater than each of their baseline tension at the concentrations less than $30 \mu \mathrm{M}$ bupivacaine. PTT's were nearly equal to each of their baseline tension at the concentrations less than 1 $\mu \mathrm{M}$ bupivacaine, and normalized TF revealed that the decay of tetanic tension began to gradually increase from $0.01 \mu \mathrm{M}$ bupivacaine (Fig. 1A).

The potencies of bupivacaine for ST and normalized TF were $50.3( \pm 5.8)$, and $11.4( \pm 1.1) \mu \mathrm{M}$, respectively. In view of the increase of normalized TF, bupivacaine interrupted neuromuscular conduction below its therapeutic concentration. The potencies of rocuronium for ST and TF were $15.5( \pm 0.4)$, and $5.6( \pm 0.4) \mu \mathrm{M}$, respectively. The potency ratios of ST to TF
A

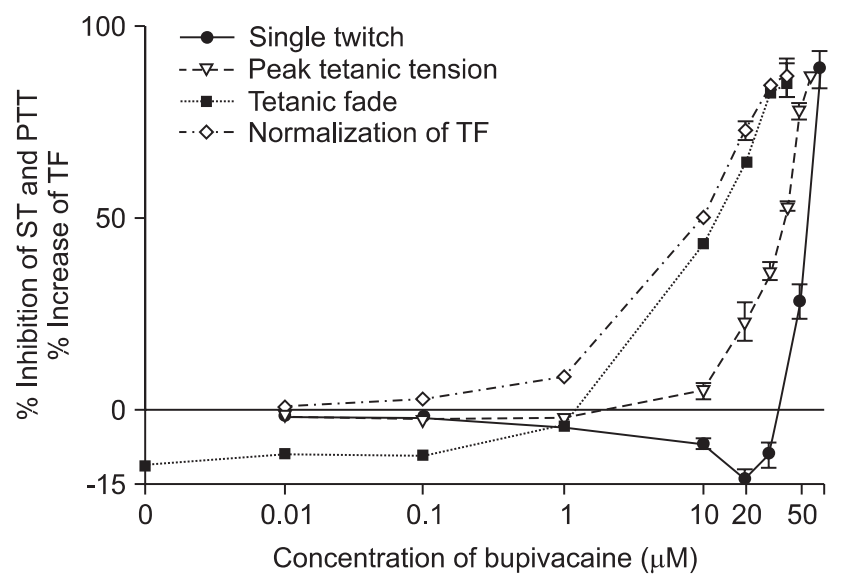

B

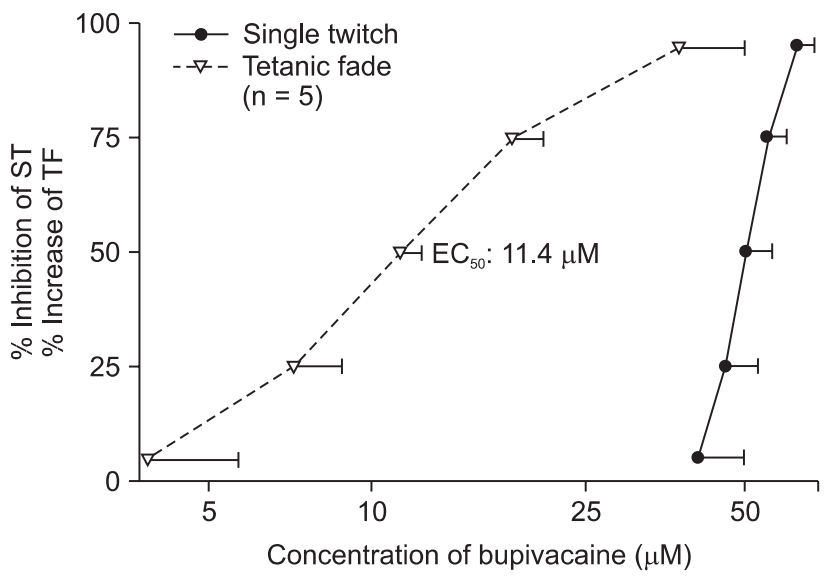

Fig. 1. Concentration-response (CR) relationship of bupivacaine. (A) shows CR curves from raw data. Tensions of ST are equal to or greater than each of their baseline tension at below $30 \mu \mathrm{M}$. PTT's are almost equal to baseline at below $1 \mu \mathrm{M}$. Normalized TF shows increases in fade from $0.01 \mu \mathrm{M}$. Values present mean and SEM. No SEM bars are shown because they are within the symbol. (B) shows CR curves from probit-derived effective CR data. $\mathrm{EC}_{50}$ 's of ST, and TF are $50.3( \pm 5.8)$, and $11.4( \pm 1.1) \mu \mathrm{M}$, respectively. Values are mean \pm SD. The scale of ordinate presents percentage and that of abscissa, $\log$ (concentration). ST: single twitch, PTT: peak tetanic tension, TF: tetanic fade.

A

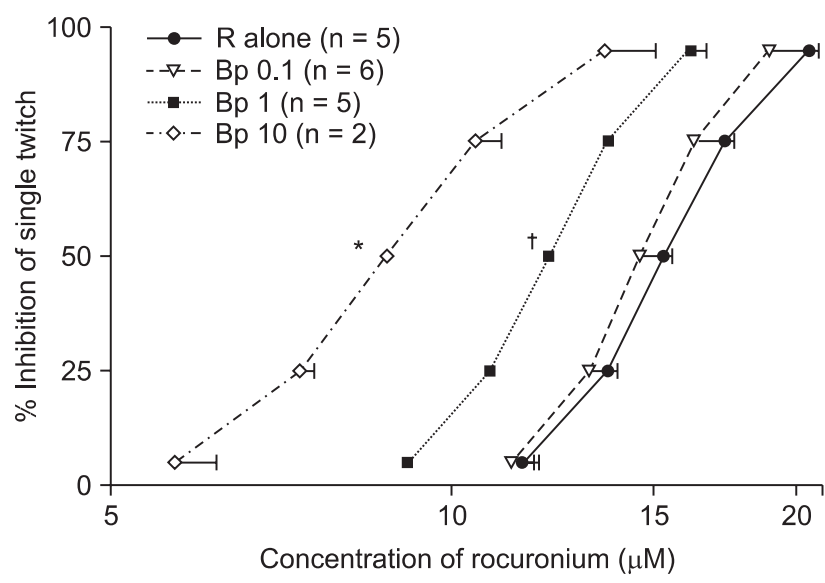

B

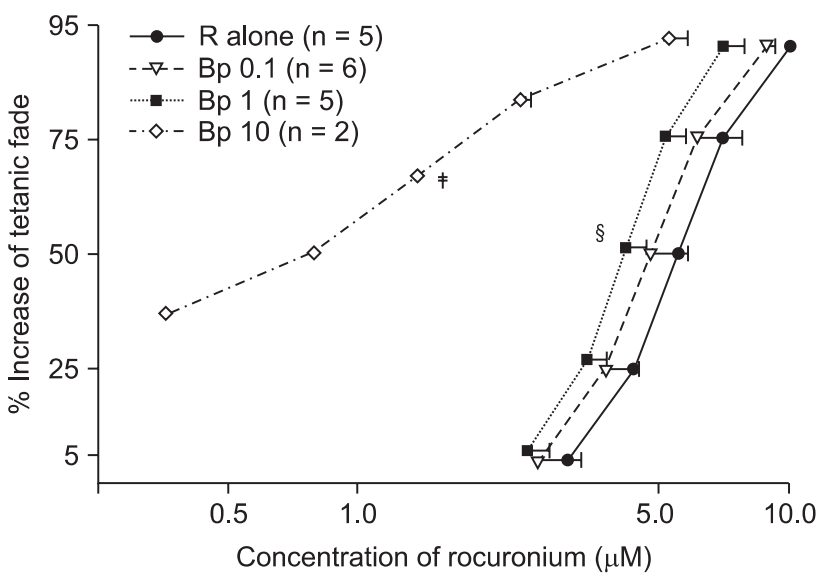

Fig. 2. In view of the cumulative concentration-effect curves for rocuronium on a ST at $0.1 \mathrm{~Hz}(\mathrm{~A})$ and $\mathrm{TF}$ at $50 \mathrm{~Hz}$ for 1.9 seconds (B), bupivacaine shifts curves to the left; hence, the drug augments the potency of rocuronium. Bupivacaine of $10 \mu \mathrm{M}$ causes $36.8 \%$ increase of $\mathrm{TF}$. The unit of concentrations for bupivacaine is $\mu \mathrm{M}$. The scale of ordinate presents percentage, and that of abscissa, log (concentration). The values of concentration are mean \pm SD. No SD bars are shown because they are within the symbol. Bp represents bupivacaine, and $\mathrm{R}$, rocuronium. * or $^{\ddagger}$ indicates $\mathrm{P}<0.05$, vs all the rest groups, ${ }^{\dagger}$ indicates $\mathrm{P}<0.05$, vs $\mathrm{R}$ alone, or $\mathrm{Bp} 0.1$, and ${ }^{\S}$ indicates $\mathrm{P}<0.05$, vs $\mathrm{R}$ alone. 
for bupivacaine and rocuronium were approximately $4.5( \pm 0.8)$ and $2.8( \pm 0.3)$, respectively $(\mathrm{P}=0.001)$ (Fig. 1B).

Bupivacaine of 1.0 and $10 \mu \mathrm{M}$ significantly shifted to left the concentration-response curve of rocuronium for ST and TF (Fig. 2). Bupivacaine of $10 \mu \mathrm{M}$ caused approximately $36.8 \%$ increase of normalized TF (Fig. 2B). The interaction indices for a ST and TF were both approximately 0.8 with bupivacaine $1.0 \mu \mathrm{M}$. Because the interaction indices were less than one, rocuronium, and bupivacaine interacted synergistically.

An amount of bupivacaine which needed to decay a tetanic tension was smaller than an amount of bupivacaine which needed to inhibit ST tension (Fig. 1 and 2).

\section{Discussion}

LAs primarily inhibit voltage-gated $\mathrm{Na}^{+}$channels [2]. However, LAs noncompetitively inhibit the ion channel activity of muscle-type nicotinic AChR at a therapeutic dose [4]. It has been known that some local anesthetics has neuromuscular block and potentiates nondepolarizing NM blockers [8-11]. Despite a commonly used LA, it still remains to investigate what effect bupivacaine has on NM conduction and rocuroniuminduced NM blockade.

Our results demonstrated that bupivacaine can inhibit NM conduction below therapeutic concentration of bupivacaine, when the decay of tetanic tension was examined $[14,15]$. Normalized TF began to increase from bupivacaine of the smallest concentration studied. If an inhibition of ST would be used as a tool to seek a NM interruption produced by bupivacaine, we would have failed to find the NM blockade induced by bupivacaine of the lower concentrations studied. Probably bupivacaine seems to produce TF in two ways: bupivacaine has a higher affinity for open state than for closed state of AChR ion channel [16-18] and may depress ACh synthesis presynaptically [1].

In addition, LA has a higher affinity for open and inactivated states of $\mathrm{Na}^{+}$channel (phasic block) than for resting (closed) states (tonic block) $[19,20]$; phasic block of $\mathrm{Na}^{+}$current by bupivacaine may block motor nerve impulses and may seem to some extent, to contribute to enhancing TF as concentrations of LA equal to those required to block nerve impulses that reduce chemically gated postjunctional currents of the nicotinic AChR $[2,4]$. Most of the drugs generate TF increase at lower concentration than do ST inhibition except for gentamicin, which causes ST reduction at lower concentration than does TF increase [12].

At the concentrations less than $30 \mu \mathrm{M}$, bupivacaine potentiated the tensions of ST above those of the baseline, even if bupivacaine would bind to and inhibit nicotinic AChR of muscle [21], and voltage gated $\mathrm{Na}^{+}$channel of motor nerve [2].
The twitch potentiation sustained despite the development of tetanic fade and partial inhibition of PTT. In view of these results, it would seem likely that bupivacaine may augment the probability of ACh release in the active zone of nerve terminal and produce the resultant twitch augmentation. Of course, this hypothesis needs to be examined with some sophisticated method, such as binomial analysis of quantal transmitter release [22].

The inhibition of ST appeared at higher concentrations of bupivacaine. LAs inhibit channel opening, increase inactive form of AChR $[4,21]$ and decrease the mean open time of ion channel of AChR [3]. In addition of its effect on NM junction, depression of motor nerve conduction would play some role in inhibiting ST [23]. Based on the dual phases of the effect of bupivacaine on ST, it seems likely that at higher concentration of bupivacaine the mechanism of ST inhibition dominated that of ST potentiation.

Elmqvist and Quastel [24] showed that the rapid decline in the quantum content of the end plate potentials seen in the beginning of tetanus can be attributed to depletion of readily releasable ACh in active zones. According to their conclusion, early part of tetanic tension (less than 10 impulses $(0.2 \mathrm{~s})$ in $50 \mathrm{~Hz}$ of tetanic stimulation) would correspond to a tension which is produced by ACh released from a store of readily releasable $\mathrm{ACh}$. So, we measured a tetanic tension at $0.2 \mathrm{~s}$ after tetanic stimulation and termed it as PTT. In our results, even though an increase of TF grew, the magnitude of PTT was equal to or greater than that of the baseline tension at the concentrations less than or equal to $1.0 \mu \mathrm{M}$ bupivacaine. Therefore, at those concentrations bupivacaine seemed to not hinder a replenishment of a store of readily releasable ACh following a tetanic stimulation. In addition, it appears likely that bupivacaine may augment the probability of ACh release and may not make the mean open time of ion channel sufficiently shorten to decrease the amplitude of PTT [3].

After extradurally injecting bupivacaine $(0.5 \%$ [20 ml], total dose: $100 \mathrm{mg}$ ), maximum concentration of bupivacaine is $1.69(0.34) \mu \mathrm{M}$, and after $90 \mathrm{~min}$, the concentration was above $0.92 \mu \mathrm{M}[14]$. The increase of normalized TF began to develop at the lowest concentration studied. This result showed that bupivacaine of a therapeutic concentration could cause NM blockade. The possible mechanisms might be considered as follows: reducing the mean open time by blocking open ion channels $[3,21]$, depressing ACh mobilization during tetanic stimulation $[1,4]$, and accelerating early excretion of stored ACh to exaggerate an increase of TF. In case of appearance of TF at lower concentration of a drug, a tetanic stimulation may be appropriate as a tool to be used for seeking NM blockade of the drug. It seems rare that a study on a contraction tension of muscle preparation has shown an inhibitory effect produced by 
LAs below its therapeutic concentration.

Potency ratio of ST to TF for bupivacaine was greater than that for rocuronium. The two drugs may have mechanisms in causing a decayed tetanic tension as follows: rocuronium occludes AChR and reduces ACh mobilization. In contrast, bupivacaine blocks open ion channels of AChR and reduces ACh synthesis. Although there is no specific known reason, the ion channel blockade by bupivacaine may be more efficient than AChR blockade by rocuronium in causing TF.

Together with its inhibition of NM transmission, bupivacaine facilitated NM blockade caused by rocuronium. Epidurally administered lidocaine, mepivacaine, and levobupivacaine delayed the recovery from vecuronium-induced NM blockade $[8,9,11,25]$. Wang et al. [10] demonstrated that procaine and lidocaine enhanced the inhibitory effect of vecuronium, and cisatracurium, at adult muscle-type nicotinic acetylcholine receptors. Therefore, like other LAs, bupivacaine may also potentiate the effect of nondepolarizing NM blockers. However, some investigators indicated that lidocaine failed to show a sparing effect on cisatracurium requirements $[6,7]$.

It has been proposed that an interaction index between 0.9 and 1.1 designates an additive interaction [26]. Thus, the interaction indices in this study indicate that rocuronium, and bupivacaine were synergistic in the NM blockade. Although it remains to be tested, several phenomena may account for the synergy of the two drugs. If one of them would be listed, mutually nonexclusive drugs contain an element of intrinsic synergy, which may contribute to the overall synergism [26]. Since they act on the different sites in AChR ion channel complex [27], rocuronium, and bupivacaine may be mutually nonexclusive and offer some degree of intrinsic synergism. Although the interaction is small, anesthesiologists should bear this interaction in mind when conducting both general and epidural anesthesia.

Normalized TF revealed NM blockade induced by bupivacaine of therapeutic concentrations. Diaphragmatic contraction for inspiration corresponds to tetanus for $2 \mathrm{~s}$, when breath rate is $10 / \mathrm{min}$, though the neuronal respiratory drive is $10-20 \mathrm{~Hz}$ during inspiration [28]. However, the physiologic frequency for a tetanic response is 30 to $50 \mathrm{~Hz}$ [29]. This has important physiologic implications because it implies that $50-\mathrm{Hz}$ tetanus is the maximum tetanus that should be applied during peripheral nerve stimulation [30]. Furthermore, $50-\mathrm{Hz}$ stimulation made a tetanic tension of baseline ascend. The increase of TF for the baseline tetanic tension had a negative value. Thus, the increase of TF in baseline tetanic tension was made zero by normalization. Based on the above findings, it appears appropriate for normalization of TF to be applied to the study on NM blockade caused by bupivacaine.

In conclusion, clinicians should be aware that at its thera- peutic concentrations, bupivacaine by itself interrupt NM conduction and thereby enhancing rocuronium-induced NM blockade.

\section{Acknowledgements}

This study was supported by research funds from Dong-A University.

\section{References}

1. Sundman E, Witt H, Olsson R, Ekberg O, Kuylenstierna R, Eriksson LI. The incidence and mechanisms of pharyngeal and upper esophageal dysfunction in partially paralyzed humans: pharyngeal videoradiography and simultaneous manometry after atracurium. Anesthesiology 2000; 92: 977-84.

2. Butterworth JF 4th, Strichartz GR. Molecular mechanisms of local anesthesia: a review. Anesthesiology 1990; 72: 711-34.

3. Neher E, Steinbach JH. Local anaesthetics transiently block currents through single acetylcholine-receptor channels. J Physiol 1978; 277: 153-76.

4. Gentry CL, Lukas RJ. Local anesthetics noncompetitively inhibit function of four distinct nicotinic acetylcholine receptor subtypes. J Pharmacol Exp Ther 2001; 299: 1038-48.

5. Fujii T, Masai M, Misawa H, Okuda T, Takada-Takatori Y, Moriwaki $\mathrm{Y}$, et al. Acetylcholine synthesis and release in NIH3T3 cells coexpressing the high-affinity choline transporter and choline acetyltransferase. J Neurosci Res 2009; 87: 3024-32.

6. Hans GA, Defresne A, Ki B, Bonhomme V, Kaba A, Legrain C, et al. Effect of an intravenous infusion of lidocaine on cisatracuriuminduced neuromuscular block duration: a randomized-controlled trial. Acta Anaesthesiol Scand 2010; 54: 1192-6.

7. Saadawy IM, Kaki AM, Abd El Latif AA, Abd-Elmaksoud AM, Tolba OM. Lidocaine vs. magnesium: effect on analgesia after a laparoscopic cholecystectomy. Acta Anaesthesiol Scand 2010; 54: 549-56.

8. Munakata K, Suzuki T, Watanabe N, Nagai H, Kakishita M, Saeki $\mathrm{S}$, et al. Influence of epidural lidocaine injection on vecuroniuminduced neuromuscular blockade. Masui 2004; 53: 1377-80.

9. Suzuki T, Mizutani H, Ishikawa K, Miyake E, Saeki S, Ogawa S. Epidurally administered mepivacaine delays recovery of train-offour ratio from vecuronium-induced neuromuscular block. Br J Anaesth 2007; 99: 721-5.

10. Wang H, Zhang Y, Li ST. The effect of local anesthetics on the inhibition of adult muscle-type nicotinic acetylcholine receptors by nondepolarizing muscle relaxants. Eur J Pharmacol 2010; 630: 2933.

11. Sahin SH, Colak A, Sezer A, Arar C, Sevdi S, Gunday I, et al. Effect of epidural levobupivacaine on recovery from vecuronium-induced neuromuscular block in patients undergoing lower abdominal surgery. Anaesth Intensive Care 2011; 39: 607-10.

12. Lee SI, Lee JH, Lee SC, Lee JM, Lee JH. Calcium and neostigmine antagonize gentamicin, but augment clindamycin-induced tetanic fade in rat phrenic nerve-hemidiaphragm preparations. J Anesth 2008; 22 : 385-90. 
13. Berenbaum MC. What is synergy? Pharmacol Rev 1989; 41: 93-141.

14. Morrison LM, Emanuelsson BM, McClure JH, Pollok AJ, McKeown DW, Brockway M, et al. Efficacy and kinetics of extradural ropivacaine: Comparison with bupivacaine. Br J Anaesth 1994; 72: 1649.

15. Cuvillon P, Nouvellon E, Ripart J, Boyer JC, Dehour L, Mahamat A, et al. A comparison of the pharmacodynamics and pharmacokinetics of bupivacaine, ropivacaine (with epinephrine) and their equal volume mixtures with lidocaine used for femoral and sciatic nerve blocks: a double-blind randomized study. Anesth Analg 2009; 108: 641-9.

16. Steinbach $\mathrm{AB}$. Alteration by xylocaine (lidocaine) and its derivatives of the time course of the end plate potential. J Gen Physiol 1968; 52: 144-61.

17. Ikeda SR, Aronstam RS, Daly JW, Aracava Y, Albuquerque EX. Interactions of bupivacaine with ionic channels of the nicotinic receptor. electrophysiological and biochemical studies. Mol Pharmacol 1984; 26: 293-303.

18. Arias HR. Role of local anesthetics on both cholinergic and serotonergic ionotropic receptors. Neurosci Biobehav Rev 1999; 23: 817 43.

19. Courtney KR. Mechanism of frequency-dependent inhibition of sodium currents in frog myelinated nerve by the lidocaine derivative GEA. J Pharmacol Exp Ther 1975; 195: 225-36.

20. Chernoff DM. Kinetic analysis of phasic inhibition of neuronal sodium currents by lidocaine and bupivacaine. Biophys J 1990; 58: 53-68.

21. Arias HR, Blanton MP. Molecular and physicochemical aspects of local anesthetics acting on nicotinic acetylcholine receptorcontaining membranes. Mini Rev Med Chem 2002; 2: 385-410.

22. Miyamoto MD. Binomial analysis of quantal transmitter release at glycerol treated frog neuromuscular junctions. J Physiol 1975; 250: 121-42.

23. Brau ME, Vogel W, Hempelmann G. Fundamental properties of local anesthetics: half-maximal blocking concentrations for tonic block of na+ and $\mathrm{K}+$ channels in peripheral nerve. Anesth Analg 1998; 87: 885-9.

24. Elmqvist D, Quastel DM. A quantitative study of end-plate potentials in isolated human muscle. J Physiol 1965; 178: 505-29.

25. Taivainen T, Meretoja OA, Rosenberg PH. The effect of epidural bupivacaine on vecuronium-induced neuromuscular blockade in children. Acta Anaesthesiol Scand 1994; 38: 453-6.

26. Chou TC. Theoretical basis, experimental design, and computerized simulation of synergism and antagonism in drug combination studies. Pharmacol Rev 2006; 58: 621-81.

27. Leonard RJ, Labarca CG, Charnet P, Davidson N, Lester HA. Evidence that the M2 membrane-spanning region lines the ion channel pore of the nicotinic receptor. Science 1988; 242: 1578-81.

28. McKenzie DK, Butler JE, Gandevia SC. Respiratory muscle function and activation in chronic obstructive pulmonary disease. J Appl Physiol 2009; 107: 621-9.

29. Merton PA. Voluntary strength and fatigue. J Physiol 1954; 123: 55364.

30. Ali HH, Savarese JJ, Lebowitz PW, Ramsey FM. Twitch, tetanus and train-of-four as indices of recovery from nondepolarizing neuromuscular blockade. Anesthesiology 1981; 54: 294-7. 